Assessing inference of the basic reproduction number in an SIR model incorporating a growth-scaling parameter

Peer-reviewed author version

GANYANI, Tapiwa; FAES, Christel; Chowell, Gerardo \& HENS, Niel (2018)

Assessing inference of the basic reproduction number in an SIR model incorporating a growth-scaling parameter. In: STATISTICS IN MEDICINE, 37 (29), p. 4490-4506.

DOI: $10.1002 / \operatorname{sim} .7935$

Handle: http://hdl.handle.net/1942/27300 


\section{Assessing inference of the basic reproduction number in an SIR model incorporating a growth-scaling parameter}

Tapiwa Ganyani ${ }^{1}$, Christel Faes ${ }^{1}$, Gerardo Chowell ${ }^{2,3}$, Niel Hens ${ }^{1,4}$

${ }^{1}$ Interuniversity Institute for Biostatistics and statistical Bioinformatics, UHasselt (Hasselt University), Diepenbeek, Belgium

${ }^{2}$ School of Public Health, Georgia State University, Atlanta, GA, USA

${ }^{3}$ Division of International Epidemiology and Population Studies, Fogarty International Center, National Institute of Health, Bethesda, MD, USA

${ }^{4}$ Centre for Health Economics Research and Modelling Infectious Diseases, Vaccine and Infectious Disease Institute, University of Antwerp, Antwerp, Belgium

\section{Corresponding author:}

Tapiwa Ganyani

Email: tapiwa.ganyani@uhasselt.be

Telephone: + 32-11-268281 


\begin{abstract}
The standard mass action, which assumes that infectious disease transmission occurs in well-mixed populations, is popular for formulating compartmental epidemic models. Compartmental epidemic models often follow standard mass action for simplicity and to gain insight into transmission dynamics as it often performs well at reproducing disease dynamics in large populations. In this work, we formulate discrete time stochastic Susceptible-Infected-Removed models with bi-linear (standard) and non-linear mass action structures to mimic varying mixing levels. Using simulations and real epidemic data, we demonstrate the sensitivity of the basic reproduction number to these mathematical structures of the force of infection. Our results suggest the need to consider non-linear mass action in order to generate more accurate estimates of the basic reproduction number although its uncertainty increases due to the addition of one growth scaling parameter.
\end{abstract}

Key words: discrete time stochastic SIR model, mass action principle, basic reproduction number, epidemic modelling, early epidemic growth phase 


\section{Introduction}

Epidemic modeling has for a long time been employed to study transmission and control mechanisms of the spread of infectious diseases. One useful parameter that quantifies the transmission potential of an infectious disease during the early take-off of an epidemic is the basic reproduction number, denoted by $R_{0}$ [1]. This quantity denotes the average number of cases generated by one infected case over the course of its infectious period, in a completely susceptible population. It is useful for its threshold property, if $R_{0} \leq 1$ then the epidemic will die out and, if $R_{0}>1$ then the epidemic can grow [2]. As such, this is a key epidemiological parameter for understanding an infectious disease' ability to invade a population that can be used to guide epidemic prevention strategies as well as for gauging the scope of public health interventions aimed at bringing the disease under control.

Reliable inferences about key transmission parameters and disease forecasts hinges on the capacity to develop a model that faithfully captures disease dynamics. The most popular epidemic models are formulated based on the assumption of population wide random mixing, where, population mixing is represented using the standard mass action principle [3]. This principle assumes that individuals within a population have an equal chance of getting infected. Denoting $S(t)$ and $I(t)$ to be the number of susceptible and infective individuals in a population at time $t$ respectively, and $\beta$ to be the infection rate, the principle says that the number of new infections is proportional to $I(t)$ and $S(t)$ and observed at the rate (incidence rate), $\frac{\beta S(t) I(t)}{N}$, where $N$ is the total population size [4].

Assuming random mixing only provides an approximation for modeling disease transmission dynamics. For instance, it may be that the contact structure of individuals within a population does not reflect that of chemical molecules in solution, i.e, it assumes that healthy and infected individuals are highly mobile, have no movement boundaries and therefore meet randomly. Although possibly unrealistic, some epidemic models that assume random mixing have performed remarkably well at reproducing disease dynamics 
particularly in large population contexts $[5,6,7]$. Despite the limitations, these models continue to provide useful insight into the transmission dynamics and control of infectious diseases [8].

Departures from standard mass action can be derived by modifying the mathematical form of the infection rate (see for example $[9,10,11])$. That is, the incidence rate is modified by introducing growth scaling exponents which aim to capture scenarios where the number of contacts that an individual has is in fact less than that obtained by the standard mass action form. Others have deviated from standard mass action by considering specific contact networks (see for example $[12,13,14,15]$ ). These models recognize that in practice, within a population, an individual has a finite number of contacts and these contacts are not necessarily distributed randomly. Network models are often mathematically and computationally complex due to high dimensionality of the networks and difficult to parameterize in the absence of detailed contact network datasets (see overview of challenges of these models in [16]).

Here we investigate compartmental epidemic models to study the sensitivity of $R_{0}$ to the mathematical form of the force of infection when the goal is to generate accurate estimates of $R_{0}$ from early case incidence data. For this purpose, we formulate a discrete time stochastic Susceptible-Infected-Removed (SIR) model (see for example [17, 18]), with an incidence rate of the form $\frac{\beta S(t) I^{\gamma}(t)}{N}$, where the power $0<\gamma \leq 1$ captures deviation from the standard mass action principle, i.e. $\gamma=1$. We begin by studying whether $\beta$ and $\gamma$ as well as other relevant parameters can be well estimated from limited data of the early epidemic growth phase. We then study the robustness of estimating $R_{0}$ in a model formulated based on the standard mass action principle $\left(\frac{\beta S(t) I(t)}{N}\right)$ on data simulated using a modified version $\left(\frac{\beta S(t) I^{\gamma}(t)}{N}\right)$. Finally we illustrate this modeling and estimation framework for estimating $R_{0}$ to three real epidemic outbreaks. 


\section{Methods}

\subsection{Model development}

\subsubsection{The SIR Model}

A simplified version of the original SIR model [19] that is commonly used assumes a closed population which is divided into three compartments, $S(t)$ - number of susceptible individuals at time $t, I(t)$ - number of infectious individuals at time $t$ that is capable of infecting susceptible individuals, and $R(t)$ - number of recovered/removed (immune or dead) individuals at time $t$. The idea behind this model is that, initially, there is a single or few infective(s); during their infectious period they transmit infection to susceptibles; the disease continues to spread in this manner until there are no more infectives. In continuous time, the SIR model is described by the following set of ordinary differential equations:

$$
\begin{aligned}
\frac{d S(t)}{d t} & =-\frac{\beta S(t) I(t)}{N} \\
\frac{d I(t)}{d t} & =\frac{\beta S(t) I(t)}{N}-\alpha I(t) \\
\frac{d R(t)}{d t} & =\alpha I(t),
\end{aligned}
$$

where $S(t)+I(t)+R(t)=N$ (population size), $\beta$ is the transmission parameter, $\alpha$ is the removal parameter and $t$ represents calendar time. The transmission parameter describes the rate at which two individuals come into effective contact per unit time and, the removal parameter describes the rate at which individuals are removed from the infected compartment. The initial condition of (1) are known positive integers $S(0), I(0)$, and $R(0)=0 . R_{0}$ is given by $\beta / \alpha$ (see for example $[20]$ ).

\subsubsection{The discrete time stochastic SIR Model}

The model described by (1) is deterministic, i.e., it ignores randomness in the manner in which individuals move from one compartment to another. This implies that the number of individuals in a compartment at a given time $t$ is uniquely determined by values 
of model parameters $(\beta$ or $\alpha)$ as well as by the number of individuals present in relevant compartments at the previous time point. In the context of small population sizes stochastic models offer a more realistic alternative to deterministic models by allowing for randomness in the epidemic process [17].

Let $N_{I}(t)$ and $N_{R}(t)$ be counting processes which denote, at time $t$, the number of individuals who have been infected and the number of individuals who have recovered, respectively. Following the work of [6], a stochastic version of (1) can be formulated as a bivariate continuous-time Markov process. For $t<h$, assuming $N_{I}(t)$ and $N_{R}(t)$ are Poisson processes, the stochastic SIR system is specified by the following infinitesimal increment probabilities,

$$
\begin{aligned}
P\left(\Delta N_{I}(t)=1 \mid N_{I}(t)\right) & =\frac{\beta S(t) I(t)}{N} h+o(h) \\
P\left(\Delta N_{R}(t)=1 \mid N_{R}(t)\right) & =\alpha I(t) h+o(h),
\end{aligned}
$$

where $\Delta N_{i}(t)=N_{i}(t+h)-N_{i}(t) i \in(I, R)$ denotes increments of $N_{i}(t)$ and, $o(h)$ tends to zero in limit as $h$ approaches zero. The increments of the counting processes are related with the state variables as,

$$
\begin{aligned}
S(t+h) & =S(t)-\Delta N_{I}(t) \\
I(t+h) & =I(t)+\Delta N_{I}(t)-\Delta N_{R}(t) \\
R(t+h) & =R(t)+\Delta N_{R}(t) .
\end{aligned}
$$

A direct consequence of assuming $N_{I}(t)$ and $N_{R}(t)$ are Poisson processes is that the amount of time until the next individual gets infected as well as the amount of time until an infected person recovers are exponentially distributed. Since $N_{I}(t)$ and $N_{R}(t)$ 
are assumed to be Poisson processes, we have that,

$$
\begin{aligned}
P\left(\Delta N_{I}(t)=n_{1}\right) & =\frac{\exp \left(-\frac{\beta S(t) I(t)}{N} h\right)\left(\frac{\beta S(t) I(t)}{N} h\right)^{n_{1}}}{n_{1} !} \\
P\left(\Delta N_{R}(t)=n_{2}\right) & =\frac{\exp (-\alpha I(t) h)(\alpha I(t) h)^{n_{2}}}{n_{2} !},
\end{aligned}
$$

or simply,

$$
\begin{aligned}
\Delta N_{I}(t) & \sim \operatorname{Po}\left(\frac{\beta S(t) I(t)}{N} h\right) \\
\Delta N_{R}(t) & \sim \operatorname{Po}(\alpha I(t) h),
\end{aligned}
$$

see for example [21]. In this way, values of model parameters as well as numbers present at the previous time points play a role in determining numbers at succeeding time points while explicitly acknowledging uncertainty in the manner in which individuals transit from one compartment to another. We refer to the model described by ( 3 and 5 ) as the discrete time stochastic SIR model. Conditional on $S(t)$ and $I(t)$ as well as on model parameters, the random variables $\Delta N_{I}(t)$ and $\Delta N_{R}(t)$ are independent.

Due to the unbounded nature of Poisson random variables, $S(t)-\Delta N_{I}(t)$ and $I(t)-$ $\Delta N_{R}(t)$ can become negative. In the case of $S(t)-\Delta N_{I}(t)$, this problem was not encountered due to the large value of $S(t)$ as well as due to the set of parameters used in the simulation analyses. For the latter, we addressed this problem by bounding $N_{R}(t)$ as,

$$
N_{R}(t)=\min (\sim \operatorname{Po}(\alpha I(t) h), I(t))
$$

The problem can also be addressed by using the Binomial distribution as in [18].

Taking expectations of (2) and (3), diving by $h$ and taking the limit as $h \rightarrow 0$ we obtain (1). Also, by the law of large numbers, for large $N,(3)$ approaches (1). Hence, the meaning of $\beta, \alpha$ and hence $R_{0}$ defined in the context of the deterministic model (1) 
carries over, approximately, to the stochastic model (3). However for the latter, there is a difference in the interpretation of $R_{0}$ - if $R_{0}>1$ it is not certain that a major epidemic will take off, in fact, a major epidemic will occur with some probability determined by model parameters [22].

In practice, epidemic data are oftentimes observed in discrete time intervals rather than continuous time (e.g. daily, weekly, monthly) and they reflect aggregated information between consecutive reporting periods. However, the reporting period, $h_{r p}$ say, is never sufficiently small in the mathematical sense (i.e. close to zero) such that (3) fairly approximates (1), moreover, it may not be fixed through the reporting period. Here, for simplicity we assume that $h=h_{r p}$ equal to one day is sufficiently small. Note that it is not necessary to make these simplifying assumptions as there are inference approaches available in the literature to address them [23].

The discrete time stochastic SIR model is a special case of the so-called time series SIR model (TSIR model) introduced by [24] and employed to model measles data assuming $h=1=h_{r p}$ time unit (bi-weekly scale) and that all infected individuals at time $t$ recover within the next $h=1$ time units. In this work we consider epidemic data available on a daily scale and assume $h=1=h_{r p}$ time units.

\subsubsection{The standard mass action principle and its modifications}

In the development of compartmental epidemic models, the mass action principle plays an important assumption since it mathematically characterizes the force of infection. The standard version implies that newly infected cases at time $t+h$ are directly proportional, jointly, to $S(t) I(t)$. This in turn implies that the incidence rate is linearly increasing with respect to the number of infectives. In [25], researchers highlighted that this assumption is unlikely to remain true when the number of infectives is large. For instance, with a large number of infectives, newly infected cases may emerge more slowly than linearly 
due to, among other reasons, clustering of infectives or reactive behavioral changes which tend to reduce the number of contacts per unit time. A combination of these mechanisms could explain slower than exponential growth dynamics during the first 3-5 disease generations for a number of disease outbreaks [26].

In light of these as well as other features that can be encountered in disease transmission, several different functional forms for incidence rates have been proposed in the literature (see overview in [27]). For instance, in [28], researchers studied bovine tuberculosis, by modifying standard mass action to the form: $\beta\left(N-\frac{[I(t)+R(t)]}{q}\right) I(t)$ with $0<q<1$ in order to account for spatial aggregation of the disease. The constant $q$, called an aggregation parameter, reduces the number of effective susceptibles per infective. It accounts for the fact that if the disease is aggregated in space, there will be a lower chance that an animal encountered by an infectious one will be susceptible. Another modification is the so called negative binomial transmission term, $S(t) \ln \left[1+\frac{\beta I(t)}{k}\right]^{k}$ with a small $k$ corresponding to highly aggregated infections, whereas $k=\infty$ reduces to standard mass action [29]. This modification reduces the force of infection or the effective number of infectives per susceptible.

A more general modification of the infection rate can be found in $[9,10,11]: \frac{\beta S^{\delta}(t) I^{\gamma}(t)}{N}$, where $0 \leq \delta, \gamma \leq 1$ are positive constants called mixing parameters. Depending on the values of $\delta$ and $\gamma$, it allows a variety of epidemic behaviors by reducing both the number of effective susceptibles per infective as well as the effective number of infectives per susceptible. As such, $\delta$ and $\gamma$ can be interpreted as describing how susceptible and infective numbers influence the generation of new cases, independently of each other. In [9] researchers provide an intuitively appealing argument for using mixing parameters a slowly growing epidemic could be due to a very small $\beta$, however, if $I(t)$ is greatly increased while incidence remains relatively low, then this could be accounted for by choosing a value of $\gamma$ less than 1 . Conversely, a rapidly growing epidemic could be due to a very large $\beta$, however, if $I(t)$ is greatly increased while incidence increases more rapidly 
than linearly, then this could be accounted for by choosing a value of $\gamma$ greater than 1 . When the number of susceptibles is very large then, a value of $\delta<1$ accounts for the possibility that the chance of an infective to get in contact with all the susceptibles may be reduced. A value of $\delta$ close to one explains less dependence on the number of susceptibles for a susceptible to become infected whereas, $\delta=1$ indicates similar dependence as in classical mass action.

In this paper we work with the more general form $[9,10,11]$, the main motivation being ease of interpretation. While the modifications used in [28, 29] can capture deviation from standard mass action, their interpretation may be less appealing in the context of human infections. For instance, in the former, it may be difficult to justify the assumption that the number of susceptible individuals scales with the sum of infected and recovered individuals. In the latter modification, in theory, the parameter $k$ reflects the degree of spatial clustering of infectives, however, spatial effects are not the only cause of deviation from standard mass action. On the other hand, with the modification of $[9,10,11]$, the mixing parameters capture non-linear effects of a subpopulation (susceptibles or infectives) without making extra assumptions about how numbers in one subpopulation scale with numbers in other subpopulations; also, the interpretation of mixing parameters is broad as they capture and reflect possible spatial and non-spatial heterogeneities.

In particular, we consider a simplified version, $\frac{\beta S(t) I^{\gamma}(t)}{N}$, hereafter referred to as non-linear mass action, where the role of $\gamma$ is to capture, approximately, effects of heterogeneous mixing. When $\gamma=1$, incidence follows exponential growth during the early epidemic growth phase in the absence of susceptible depletion while, $0<\gamma<1$ supports early subexponential growth dynamics [30]. In [11], it is established that the wide range of dynamic behaviors induced by this incidence rate are mainly determined by $\beta$ and $\gamma$, and to a lesser extent by $\delta$. As such, it is reasonable to expect that this simplification will, to a large extent, allow to capture disease transmission non-linearities in real life settings. We support this reasoning by noting that raising a number to a power less than one has smaller 
impact on a larger number $(S(t))$ compared to a lower number $(I(t))$, as such, fixing $\delta=1$ appears reasonable. Moreover, when it comes to model estimation it is to be expected that estimating both $\delta$ and $\gamma$ can lead to identifiability issues.

For purposes of illustration, we also consider the standard mass action principle, $\frac{\beta S(t) I(t)}{N}$, hereafter referred to as bi-linear mass action. Note that the terminology bi-linear/nonlinear mass action indicates that incidence rate is bi-linear/non-linear in $S(t)$ and $I(t)$ respectively.

\subsection{Model estimation}

As in [18], the Bayesian Markov chain Monte Carlo (MCMC) framework is used for estimating model parameters. This approach is chosen mainly for its ability to deal with the typically unobserved process, $\Delta N_{R}(t)$, by averaging over its probability distribution; this way the uncertainty of the unobserved process is properly taken into account (see for example $[18,31,32])$. Let $\Theta=\{\beta, \gamma, \alpha\}$ denote the vector of all model parameters to be estimated. Given initial conditions $\{S(0)=N-I(0), I(0)=1, R(0)=0\}$, when both $\Delta N_{I}(t)$ and $\Delta N_{R}(t)$ are observed at fixed time intervals of length $h$ then, the state variables $\{S(t), I(t)\}$ are completely determined by (3). Due to conditional independence of $\Delta N_{I}(t)$ and $\Delta N_{R}(t)$, the likelihood, $\mathcal{L}$, is given by:

$$
\mathcal{L}\left(\Delta N_{I}, \Delta N_{R} \mid \Theta\right)=\prod_{t=0}^{T} \mathrm{~g}_{I}\left(\Delta N_{I}(t) \mid \cdot\right) \mathrm{g}_{R}\left(\Delta N_{R}(t) \mid \cdot\right)
$$

where $g_{I}$ and $g_{R}$ represent the Poisson densities (5) conditioned on $\Theta$ as well as on all information $\{S(t), I(t)\}$ up to time $T$. Assigning independent priors to each of the parameters, the posterior distribution of $\Theta$ is given by:

$$
P\left(\Theta \mid \Delta N_{I}(t), \Delta N_{R}(t)\right) \propto \mathcal{L}\left(\Delta N_{I}(t), \Delta N_{R}(t) \mid \Theta\right) p(\Theta)
$$

where, $p(\Theta)$ is a product of prior distributions for $\{\beta, \gamma, \alpha\}$. The corresponding MCMC 
algorithm proceeds as follows:

1. initialize $\Theta$;

2. update $\Theta$ from $\Theta \mid \Delta N_{I}(t), \Delta N_{R}(t)$;

3. repeat step (2) until the chain has converged.

When only $\Delta N_{I}(t)$ is observed, unobserved data $\Delta N_{R}(t)$ are imputed and the posterior distribution is given by:

$$
P\left(\Theta, \Delta N_{R}(t) \mid \Delta N_{I}(t)\right) \propto \mathcal{L}\left(\Delta N_{I}(t), \Delta N_{R}(t) \mid \Theta\right) p(\Theta)
$$

where in this case, $\mathcal{L}$ is the augmented likelihood and $p(\Theta)$ is as before. The MCMC algorithm proceeds in the following steps:

1. initialize the unobserved vector $\Delta N_{R}(t)$ and construct the series $\{S(t), I(t)\}$ using $(3)$;

2. initialize $\Theta$;

3. update $\Delta N_{R}(t)$ from $\Delta N_{R}(t) \mid \Delta N_{I}(t), \Theta$ and construct the series $\{S(t), I(t)\}$ using $(3)$;

4. update $\Theta$ from $\Theta \mid \Delta N_{I}(t), \Delta N_{R}(t)$;

5. repeat step (3) and (4) until the chain has converged.

Note that $R_{0}=\beta / \alpha$ is monitored within the MCMC chain, thus, we obtain its posterior distribution from which summary statistics can be calculated. In both simulation studies and real outbreak data applications (Sections 3.1 and 3.2), minimally informative priors are specified for $\beta$ and $\gamma$. For $\alpha$, minimally informative and informative priors are used.

The posterior distribution is evaluated in OpenBUGS [33]; the code is provided in Appendix D. Though the Bayesian framework enables carrying out inference given partial data, the described MCMC algorithm may not be efficient since it does not examine 
whether proposed values of the unobserved process $\Delta N_{R}(t)$ are consistent with the size of the observed epidemic until time $t$. A modified algorithm which addresses this issue was proposed in [18]. However, this modification has to be implemented outside the OpenBUGS environment where the user can define own sampling rules.

\subsection{Simulation study}

We simulate multiple datasets incorporating effects of noise based on the discrete time stochastic SIR model assuming $h=h_{r p}=1$ day, where model parameters are fixed to some true values and then, fit model (3) to each simulated dataset. The simulation study is based on epidemics that take off and resemble a uni-modal shape similar to the deterministic model (1) (see Section 3.1).

Using simulated data we evaluate the performance of the estimation procedure. This step involves exploring the sensitivity of the estimation procedure to the choice of prior distributions as well as starting values.

When fitting dynamic non-linear models to data, it may be that parameters cannot be estimated uniquely [34], in such a case, different combinations of model parameters lead to a similar fit. When non-linear mass action is assumed, it may well be that the same epidemic curve can be described by, on the one hand, a small value of $\beta$ and a large value of $\gamma$ and, on the other hand, a large value of $\beta$ and a small value of $\gamma$. Therefore, another goal is to study whether parameters can be uniquely estimated and their uncertainty. In this work, we examine pairwise correlations from posterior samples of parameters since the pairwise relationship appeared fairly linear. Note that in order to capture non-linear dependencies between variables, a more general measure is that of mutual information $[35]$.

Moreover, since real outbreak data often does not include all SIR states, it may well be that information required to estimate some parameters is not contained in the observed 
states [18]. As such, another goal is to note whether or not posterior estimates are, to a large extent, a reflection of information contained in the prior distributions; this is checked by inspecting trace plots and systematically comparing prior and posterior estimates of each parameter.

\section{Results}

\subsection{Simulation study}

For our simulation studies, we set initial conditions and parameters as follows: $N=$ 70,000, $T=20$ days, $\beta=1.1, \alpha=0.4467, I(0)=1$ corresponding to $R_{0}=2.46$. We analyze 6 scenarios with different growth scaling parameters, $\gamma=\{0.90,0.92,0.94$, $0.96,0.98$ and 1.00$\}$. The value of $\beta$ is chosen to be in the region of estimates which were obtained from preliminary real outbreak analyses. Values of $\gamma$ are chosen $\leq 1$ to deviate from the standard bi-linear mass action assumption yielding early exponential growth scenario as well as slower than exponential growth scenarios (Appendix A); for the considered range, the sensitivity of $R_{0}$ to the mathematical structure of the force of infection is apparent. The value of $\alpha$ is chosen to model an average infectious period of about 2.2 days. Holding $\beta$ and $\gamma$ constant, the impact of $\alpha$ on the epidemic curve is that, longer average infectious periods result in larger epidemics and hence, earlier peak times as well as extinction times (see Appendix A). For each scenario, a total of 250 datasets are simulated.

[Figure 1 about here.]

We evaluate the posterior distribution across simulated datasets using 15,000 values with a thinning of 25 . On summarizing the distribution, a burn-in of 5,000 iterations is removed. The remaining 10,000 samples used for analyses appear to have converged. 
The rationale for using data up to day 20 is that $R_{0}$ is defined in the context of a completely susceptible population, i.e., in the absence of behavioral changes or interventions. In real life, as an epidemic progresses the population does not remain completely susceptible due to infection, behavioral changes and intervention measures which mitigate the transmission rate, as such, $R_{0}$ is estimated from the early onset of the epidemic. Note that there was no theoretical motivation for fixing the cut-off at day 20, nevertheless, it is to be expected that considering longer phases (using more data) may improve the estimation in terms of bias and precision, and vice-versa.

\subsubsection{How well are model parameters estimated?}

The extent to which model parameters were well estimated is studied by inspecting coverage, bias and precision. We start by investigating whether all model parameters (and hence $R_{0}$ ) can be well estimated simultaneously. The idea is to compare the estimation when only $\Delta N_{I}(t)$ is observed to the case where both $\Delta N_{I}(t)$ and $\Delta N_{R}(t)$ data are observed. In the former, minimally informative priors are assigned to $\beta$ and $\gamma$, i.e. $U(0.1,10)$ for each parameter and, for $\alpha$, a minimally informative prior is assigned i.e. $U(0.1,1)$; an informative prior is also assigned i.e. $U(0.4,0.5)$ and; the parameter is also fixed to the true value. In the latter, only the minimally informative priors are assigned to all parameters. The results are shown in Table 1.

[Table 1 about here.]

Generally, whether or not $\Delta N_{R}(t)$ is observed, $\beta$ and $\gamma$ are estimated reasonably well within the range of their true values as is seen from the magnitude of bias and standard errors as well as coverage. On the other hand, comparing the two results where $\alpha$ is assigned a minimally informative prior, $U(0.1,1)$, the results indicate that the estimation of $\alpha$ is greatly affected by the absence of $\Delta N_{R}(t)$ as is seen from an increase in bias and variability of $\alpha$ and consequently in $R_{0}$. This is not surprising because the component of the likelihood involving the parameter $\alpha$ depends only on the unobserved $\Delta N_{R}(t)$. 
As a result, a posteriori, $\alpha$ largely reflects its prior distribution thus increasing bias and variability of $R_{0}$. However, when $\alpha$ is assigned an informative prior or fixed to the true value, bias and variability in $R_{0}$ are about the same compared to the case where $\Delta N_{R}(t)$ is observed. As such, in the absence of $\Delta N_{R}(t)$, it appears justifiable to either assign $\alpha$ an informative prior or fix it to the true value.

Table 2 presents results for all different values of $\gamma$ considered while the parameter $\alpha$ is fixed to the true value. In all cases, as before, $\beta$ and $\gamma$ as well as $R_{0}$ are estimated reasonably well within the range of their true values as is seen from the magnitude of bias and standard errors as well as coverage which is above $95 \%$ for all scenarios. It is worth noting that in general, bias and variability decrease as $\gamma$ increases. This is to be expected since more data improves estimation in terms of bias and precision.

[Table 2 about here.]

Figure 2 summarizes pairwise correlations between the posterior samples of $\beta$ and $\gamma$ obtained from one of the 250 repeated simulations for two scenarios $(\gamma=0.9$ and $\gamma=1)$. Strong negative correlation is observed between $\beta$ and $\gamma$; a low value of $\beta$ corresponds to a high value of $\gamma$ and vice versa. Intuitively this is expected since similar epidemic curves can be constructed by taking, on the one hand, a low value of $\beta$ and a high value of $\gamma$, or on the other hand, by taking a high value of $\beta$ and a low value $\gamma$. Although the true parameter values are well recovered, it is difficult to estimate these two parameters separately. This can lead to small bias in the estimation of $\beta$ and, hence in the calculation of $R_{0}$ as observed in Tables 1 and 2. Also, it can lead to loss of precision since each value of $\gamma$ corresponds to a specific region of $\beta$ values, and the specific regions vary widely depending on the value (uncertainty) of $\gamma$. As such, we can expect that credible intervals of $\beta$, and hence of $R_{0}$, will become wide when $\beta$ and $\gamma$ are estimated together using non-linear mass action formulation.

[Figure 2 about here.] 


\subsubsection{Impact of mass action mis-specification}

In this section we investigate the impact of mis-specifying the mass action term modeling the force of infection. In particular, we assume that the true underlying mass action is non-linear, however, when fitting the model we specify bi-linear mass action, i.e. we naively fix $\gamma=1$. The removal rate $\alpha$ is fixed to the true value. The results are presented in Table 3.

\section{[Table 3 about here.]}

Results indicate that estimation of $\beta$ and hence of $R_{0}$ is sensitive to mis-specification of the force of infection, i.e., when the underlying mass action is non-linear, assuming bi-linear mass action has a large impact on the estimates. Extremely low coverage and severe bias are observed for $\beta$. The reason being that the model is mis-specified, a true value of $\gamma<1$ results in a slowly growing epidemic and hence, when $\gamma$ is omitted from the model, $\beta$ hides this mis-pecification by biasing downwards, hence the negative bias observed. This in turn affects the calculation of $R_{0}$, which is underestimated.

However, if focusing on precision of the estimates, it turns out that bi-linear mass action yields considerably smaller standard errors compared to non-linear mass action for both the case when the model is mis-specified (i.e. when true $\gamma=0.90,0.92,0.94,0.96,0.98$ ) as well as for the case when the true value is $\gamma=1$. This is expected since $\beta$ and $\gamma$ are highly correlated; it follows from Figure 2 that, regardless of the true value of $\gamma$, if $\gamma$ is fixed, $\beta$ values become restricted to a narrow range. Thus, when bi-linear mass action is assumed (i.e. fixing $\gamma=1$ ), narrow credible intervals will be obtained, however, they do not necessarily indicate that estimates are closer to their true values.

Focusing on the predictive ability to estimate the epidemic curve, it turns out that the mis-specified model can compensate for the omitted $\gamma$ parameter ensuring that a fairly good fit to the data can still be obtained (Figure 3). However, in terms of MSPE the fit 
does not outperform the one obtained when $\gamma$ is included in the model (Tables 2, 3 and Figure 3).

[Figure 3 about here.]

In conclusion, simulation studies show that $\beta$ and $\gamma$, and hence $R_{0}$, can be estimated fairly well within range of their true values using data of the early epidemic growth phase if the non-linear mass action model is used. When the true underlying mass action is non-linear, bi-linear mass action can lead to underestimation of $R_{0}$ although it yields an acceptable fit.

\subsection{Applications to real outbreak data}

We fitted Model (3) to incidence data of the early epidemic growth phase. In practice, gauging the period during which the epidemic grows "freely" (i.e. in the absence of intervention measures) is a non-trivial task which requires field data about the time at which intervention measures are put in place - such data are usually unavailable or are available but subjective. In the absence of this information, ad-hoc approaches were used to select the length of the early growth phase. A comparison of estimates obtained here and those obtained in previous studies is beyond the scope of this paper, as we aim to highlight potential differences in estimates obtained assuming bi-linear vs. non-linear mass action.

\subsubsection{Influenza Epidemic in the city of San Fransisco, California, 1918 to 1919}

The city of San Francisco is located in Northern California and covers a land area of about $121 \mathrm{~km}^{2}$. This city was significantly affected by the 1918 influenza pandemic. At that time, it is estimated that the city had a population of about 550,000 [36], and 28,310 cases were recorded over a period of 63 days between September and November [37]. 
The data, obtained from [37], are available in the form of newly infected cases for the September - November influenza wave. In the analyses presented in this section, case data up to day 17 of the epidemic were used. Since $\Delta N_{R}(t)$ is not observed the model is fitted with the removal rate assigned an informative prior, $U(1 / 6,1 / 3)$ i.e., average infectious period of 3-6 days [38]. Table 4 shows the results.

[Table 4 about here.]

Larger $\beta$ values are obtained with non-linear mass action, consequently, $R_{0}$ estimates are markedly larger too. Compared with simulation study results, these findings seemingly suggest that during the initial phase, the epidemic had a potential to grow fast, however, due to inhomogeneous population mixing, behavior changes or a combination of factors, it grew at a slower pace than what it would have if the population mixed homogeneously, in other words, the effective reproduction number decays rapidly. As in simulations, the decrease of $\beta$ in the bi-linear mass action is the result of the model compensating for the omitted $\gamma$ consequently leading to a smaller $R_{0}$. Also, $R_{0}$ exhibits large uncertainty when estimated using the model with non-linear mass action - this is expected as the correlation between $\beta$ and $\gamma$ induces uncertainty (section 3.1.1). While the model based on bi-linear mass action fits reasonably well it did not outperform the one based on non-linear mass action (Table 4; Figure 4).

[Figure 4 about here.]

\subsubsection{Ebola outbreak in Congo, 1976}

In 1976 Congo experienced an outbreak of Ebola, the outbreak was concentrated in the north-west part of the country in the Bumba Zone. At that time the zone had a population of about 275,000. The index case was observed in Yambuku village and most of the infected cases were recorded within a radius of $70 \mathrm{~km}$ of the village. About 318 cases 
resulting in 280 deaths were reported over a period of about 2 months [39].

The outbreak data are available in the form of daily number of newly infected cases [40, 41]. Note that though the SIR model is not best suited for diseases with a long incubation period such as Ebola, we use it for illustration purposes. We use case data up to day 19 of the epidemic. Since $\Delta N_{R}(t)$ is not observed, the model is fitted with the removal rate assigned an informative prior, $\mathrm{U}(1 / 6,1 / 5)$, i.e, average infectious period of about 5-6 days. Table 5 shows the results.

[Table 5 about here.]

Similar findings are observed as before, more importantly, smaller $R_{0}$ estimates are obtained for bi-linear mass action compared to non-linear. In terms of goodness of fit, the model based on bi-linear mass action yields a good fit though the fit is slightly poor compared to the one based on non-linear mass action (Table 5; Figure 5).

[Figure 5 about here.]

\subsubsection{Common cold outbreak on the island Tristan da Cunha, 1967}

Tristan da Cunha is an island in the south Atlantic Ocean covering a land area of $98 \mathrm{~km}^{2}$. It was observed that epidemics, such as common cold, occur on the island after arrival of ships from Cape Town. Data from a common cold outbreak that occurred over a period of 21 days in 1967 are available in [42, 43]. At the time of the outbreak the island had a population of approximately 300 inhabitants [42].

The data are available in the form of $I(t)$ and $R(t)$. In order to fit the discrete time stochastic SIR model, since the population size is known, we reconstruct $\Delta N_{I}(t)$ and $\Delta N_{R}(t)$. We use case data up to day 11 of the epidemic; Table 6 shows parameter estimates obtained. Note that for these data a minimally informative prior is used for $\alpha$ 
since simulation studies showed that $\alpha$ can be reliably estimated when data on removed cases are available (Table 1$)$.

[Table 6 about here.]

As before, smaller $\beta$ estimates are observed for bi-linear mass action compared to nonlinear, as a result, smaller $R_{0}$ estimates are obtained. Also, in terms of goodness of fit, the model based on bi-linear mass action fits acceptably good though not outperforming the one based on non-linear mass action (Table 6; Figure 6).

[Figure 6 about here.] 


\section{Discussion and Conclusions}

The sensitivity of $R_{0}$ to model assumptions has been investigated in prior studies (see for example $[44,45])$. Here, we demonstrate the sensitivity of $R_{0}$ to the mathematical form of the force of infection when it is estimated from incidence data of the early epidemic growth phase. We formulate a discrete time stochastic SIR model $[17,18]$ with non-linear mass action $[9,10,11]$. Since non-linear mass action introduces an extra parameter on the classical bi-linear mass action incidence rate, we begin by studying how well this extra parameter as well as other model parameters can be estimated from early incidence data. Thereafter, we use simulated and real epidemic data to demonstrate how the choice between non-linear and bi-linear mass action impacts the estimation of $R_{0}$.

Our simulation studies indicate that $\beta$ and $\gamma$ can be estimated altogether from the early epidemic phase with relatively low bias and variance. However, the removal rate $\alpha$ cannot be reliably estimated in the absence of data on removed individuals, in which case its estimation is largely informed by the prior distribution (Table 1). Results indicate that more accurate inference on $\beta$ and $\gamma$ (and hence on $R_{0}$ ) can however be achieved when $\alpha$ is fixed to the true value a priori, or, when assigned a prior distribution centered about the true value. In practice, information about $\alpha$ is usually available from the literature, therefore, it can be utilized to obtain more accurate $R_{0}$ estimates. On the other hand, for non-linear mass action, a high correlation is observed between $\beta$ and $\gamma$. This leads to wider credible intervals in our estimates. Nevertheless, it should be noted that narrower credible intervals obtained when bi-linear mass action is assumed are not necessarily an indication of closeness to the truth. The high correlation may also raise concerns about the interpretability of $\beta$ and $\gamma$, individually. Nevertheless, the two parameters are estimated considerably well within range of their true values and therefore, reasonable inferences can still be made.

With regards to structure of the force of infection, simulation results indicate that $R_{0}$ is sensitive to the type of mass action assumed (Tables 2 and 3). It is observed that 
when $\gamma<1$, assuming bi-linear mass action leads to severe underestimation of $R_{0}$ even though it yields an acceptably good fit (Figure 3). Assuming bi-linear mass action in the analysis of real outbreak data leads to results well in-line with simulation findings - in comparison with non-linear mass action, smaller $R_{0}$ estimates are obtained for bi-linear mass action, (Tables 4, 5 and 6). Moreover, bi-linear mass action results in acceptably good fit which however does not outperform that of non-linear mass action - the difference is small though and MPSE does not penalize for the additional parameter used in the non-linear mass action formulation (Figures 4 - 6).

Previous studies have employed non-linear incidence rates in modeling human infectious diseases (see for example $[24,46,47,48]$ ). In particular, the time-series SIR (TSIR) model has been employed to study measles dynamics. By incorporating non-homogeneous mixing using mixing parameters, their analyses are able to more accurately capture endemic cycles and episodic outbreaks in measles [49]. Our work differs from [24, 46, 47, 48] in that we study the sensitivity of $R_{0}$ to the chosen form of mass action whereas, the above studies employed non-linear mass action to capture non-linearities in disease transmission and reproduce observed dynamics in measles data. Moreover, we focus on the dynamics of the early growth phase, whereas the above studies explore long-term epidemic dynamics.

In [28], researchers study bovine tuberculosis using "mixed population" models based on a negative binomial transmission term. They also find out that accounting for heterogeneous mixing by modifying bi-linear mass action allows a higher value for the transmission parameter and consequently, a higher $R_{0}$. Nevertheless, this message has not gained traction as far as human infectious disease modeling using "mixed population" is concerned. This is evidenced by a large number of publications where such models are formulated based on bi-linear mass action. However, recently, [50] proposed a new quantity called the "empirically adjusted reproductive number". It is demonstrated that the quantity, unlike the traditional reproduction number, takes into account non-linear effects in population 
mixing and can be expected to be larger for active epidemics. In this study, non-linear effects are accounted for by conditioning on the number of contacts between a person and other individuals in the population.

Recent research has highlighted that early sub-exponential (e.g., polynomial) growth dynamics is a common phenomenon across a range of infectious disease outbreaks including influenza, Ebola, foot-and-mouth disease, HIV/AIDS, plague, measles and smallpox [26]. While our focus here is on estimating the basic reproduction number, $R_{0}$, that characterizes transmission potential at the onset of the epidemic, the effective reproduction number, $R_{t}$, is another useful quantity to monitor temporal changes in transmission potential during the course of an outbreak. In particular, $R_{t}$ remains invariant during the early growth phase of an epidemic characterized by early exponential growth dynamics whereas in the context of sub-exponential growth epidemics, $R_{t}$ declines over disease generations asymptotically towards the epidemic threshold of 1.0 [30]. This decline in $R_{t}$ is more pronounced for sub-exponential growth dynamics. Hence, our work here on estimating key transmission parameters based on a flexible SIR framework incorporating the possibility of slower than exponential epidemic growth is timely. At the same time, these observations underscore the importance of understanding the underlying mechanisms behind early polynomial growth dynamics, which could include reactive behavioral changes and the role of spatial heterogeneity.

It is worth pointing out that the SIR model we use in this work could be further improved to incorporate more realistic assumptions about the biological processes that are thought to be behind the transmission dynamics of an infectious disease. In particular, this model embeds several simplifying assumptions which may be invalid in practice and hence possibly lead to inaccurate estimates of $R_{0}$. For instance, it ignores the latent period, assumes exponentially distributed waiting times as well as a constant transmission rate. In [44], it is shown that ignoring the latent period or assuming exponentially distributed latent and infectious periods always leads to underestimation of $R_{0}$. Here we 
highlight that different assumptions in incidence rates can lead to substantially different $R_{0}$ estimates. An important extension is to investigate the impact of incorporating more realistic assumptions in the context of non-linear mass action based "mixed population" models.

Though the non-linear mass action based SIR model is rather simplistic, it explains observed epidemic data fairly well and, since the role of its parameters is clearly understood it is useful in several regards. For instance, since it allows disentangling disease transmissibility and population interaction, through $\beta$ and $\gamma$ respectively, it can be used to evaluate model complexity in general, providing insights as to whether deviation from bi-linear mass action is necessary. In the same vein, it can also be used to investigate the phenomenon of sub-exponential growth by testing the hypothesis, $H_{0}: \gamma=1$.

Another limitation of our results, particularly the simulation studies, could arise from employing a model that ignores over-dispersion; a phenomenon which could arise due to stochastic effects which are unpredictable or insufficiently understood or non-measurable [51]. As such, early sub-exponential growth could arise due to the interplay between stochasticity and deterministic effects capturing contact structure of the population rather than due to deterministic effects alone [52]. It could also arise due to other unknown and/or unmodeled processes (see for example [51] and [52]). Nevertheless, our simulation results demonstrate the sensitivity of $R_{0}$ estimates to the form of mass action, they underscore the importance of considering deviating from bi-linear mass action when the goal is to generate a reliable estimate of the transmission parameter and hence, of $R_{0}$. Sensitivity analysis of real-life outbreaks showed no evidence of over-dispersion (Appendix C), nonetheless, short time series are unlikely to reveal sufficient information to separate stochasticity and the underlying dynamics [53, 54].

Another potential source of bias in our results, particularly in the real life data sets, is the assumption that $h=h_{r p}=1$ day is sufficiently small for (3) to provide a fairly accurate 
approximation of (1). Note that this assumption is not a concern in the simulation studies since data are generated assuming $h=h_{r p}=1$ day. Sensitivity analyses performed in the inference framework for partially observed Markov processes [23] choosing $h$ to be small $(h=0.01)$ and different from $h_{r p}$ show that parameter estimates do not differ substantially (see Appendix C).

Overall, our results demonstrate using simulations and real epidemic data the sensitivity of $R_{0}$ to the mathematical structure of the force of infection, e.g., bi-linear vs. non-linear mass action. In this regard, our results demonstrate that, in comparison to non-linear mass action, a good fitting SIR model based on bi-linear mass action may not guarantee a reliable $R_{0}$ estimate. Since bi-linear mass action is a sub-case of non-linear mass action, we emphasize that the latter is worthwhile and should be considered for its ability to yield more reliable $R_{0}$ estimates as well as for its inherent property of revealing deviation from the classical bi-linear mass action assumption. In fact, when bi-linear mass action is a good approximation, inferences based on the non-linear mass action formulation allow data to speak for itself. 


\section{References}

[1] Diekmann O, Heesterbeek J, Roberts M. The construction of next-generation matrices for compartmental epidemic models. J R Soc Interface. 2009;7(47):873-885. doi:10.1098/rsif.2009.0386.

[2] Diekmann O, Heesterbeek J, Metz J. On the definition and the computation of the basic reproduction ratio $R_{0}$ in models for infectious diseases in heterogeneous populations. J Math Biol. 1990;28(4): 365-382. doi:10.1007/bf00178324.

[3] Heesterbeek H. The law of mass-action in epidemiology: a historical perspective. Ecological paradigms lost: routes of theory change. 2005;81-104. doi:10.1016/b978012088459-9/50007-8.

[4] Keeling MJ, Rohani P. Modeling infectious diseases in humans and animals. New Jersey, USA: Princeton University Press; 2008.

[5] Bailey NTJ. Some problems in the statistical analysis of epidemic data. J R Stat Soc Series B Stat Methodol. 1955;17:35-68.

[6] Bartlett MS. Stochastic population models in ecology and epidemiology, Methuen's monographs on applied probability and statistics, volume 4. London, UK: Spottiswoode, Ballantyne and Company Limited; 1960.

[7] Fine PEM, Clarkson JA. Measles in England and Wales-I: an analysis of factors underlying seasonal patterns. Int J Epidemiol. 1982;11:5-14. doi:10.1093/ije/11.1.5.

[8] Brauer F. Mathematical epidemiology: Past, present, and future. Infect Dis Model. 2017;2(2):113-127. doi:10.1016/j.idm.2017.02.001.

[9] Severo NC. Generalizations of some stochastic epidemic models. Math Biosci. 1969;4:395-402. doi:10.1016/0025-5564(69)90019-4.

[10] Liu WM, Levin SA, Isawa Y. Influence of non-linear incidence rates upon the behaviour of SIRS epidemiological models. J Math Biol. 1986;23:187-204. doi:10.1007/bf00276956. 
[11] Liu WM, Hethcote HW, Levin SA. Dynamical behavior of epidemiological models with nonlinear incidence rates. J Math Biol. 1987;25:359-380. doi:10.1007/bf00277162.

[12] Andersson H. Epidemic models and social networks. Mathematical Scientist. $1999 ; 24(2): 128-147$.

[13] Newman ME. Spread of epidemic disease on networks. Physical review E. 2002;66(1):016128. doi:10.1103/physreve.66.016128.

[14] Keeling MJ, Eames KT. Networks and epidemic models. J R Soc Interface. 2005;2(4):295-307. doi:10.1098/rsif.2005.0051.

[15] Danon L, Ford AP, House T, et al. Networks and the epidemiology of infectious disease. Interdiscip Perspect Infect Dis. 2011;2011:1-28. doi:10.1155/2011/284909.

[16] Pellis L, Ball F, Bansal S, et al. Eight challenges for network epidemic models. Epidemics. 2015;10:58-62. doi:10.1016/j.epidem.2014.07.003.

[17] Daley DJ, Gani J. Epidemic modelling: an introduction. New York, USA: Cambridge University Press; 1999.

[18] Lekone PE, Finkenstädt BF. Statistical inference in a stochastic epidemic SEIR model with control intervention: Ebola as a case study. Biometrics. 2006;62:11701177. doi:10.1111/j.1541-0420.2006.00609.x.

[19] Kermack WO, McKendrick AG. Contributions to the mathematical theory of epidemics. Part I. Proc R Soc Lond A Math Phys Sci. 1927;115:700-721. doi:10.1098/rspa.1927.0118.

[20] Anderson RM, May RM. Infectious diseases of humans: dynamics and control. Oxford, UK: Oxford university press; 1992.

[21] Ross S. Introduction to probability models eleventh edition. Oxford, UK: Elsevier; 2014. 
[22] Andersson H, Britton T. Stochastic epidemic models and their statistical analysis. Springer Science and Business Media; 2012.

[23] King AA, Nguyen D, Ionides EL. Statistical inference for partially observed Markov processes via the $\mathrm{R}$ package pomp. J Stat Softw. 2016;69(12). doi:10.18637/jss.v069.i12.

[24] Finkenstädt BF, Grenfell BT. Time series modelling of childhood diseases: a dynamical systems approach. Appl Stat. 2000;49:187-205. doi:10.1111/1467-9876.00187.

[25] Capasso V, Serio G. A generalisation of the Kermack-McKendrick deterministic epidemic model. Math Biosci. 1978;42:43-61. doi:10.1016/0025-5564(78)90006-8.

[26] Viboud C, Simonsen L, Chowell G. A generalized-growth model to characterize the early ascending phase of infectious disease outbreaks. Epidemics. 2016;15:27-37. doi:10.1016/j.epidem.2016.01.002.

[27] McCallum H, Barlow N, Hone J. How should pathogen transmission be modelled? Trends Ecol Evol. 2001;16(6):295-300. doi:10.1016/s0169-5347(01)02144-9.

[28] Barlow N. Non-linear transmission and simple models for bovine tuberculosis. J Anim Ecol. 2000;69(4):703-713. doi:10.1046/j.1365-2656.2000.00428.x.

[29] May RM. Host-parasitoid systems in patchy environments: a phenomenological model. J Anim Ecol. 1978;47(3):833-844. doi:10.2307/3674.

[30] Chowell G, Viboud C, Simonsen L, et al. Characterizing the reproduction number of epidemics with early sub-exponential growth dynamics. J R Soc Interface. 2016;13:20160659. doi:10.1098/rsif.2016.0659.

[31] Lesaffre E, Lawson AB. Bayesian Biostatistics. West Sussex, UK: John Wiley and Sons; 2012.

[32] Gelman A, Carlin J, Stern H, et al. Bayesian Data Analysis. Boca Raton, USA: Chapman and Hall/CRC; 2014. 
[33] Lunn D, Spiegelhalter DJ, Thomas A, et al. The BUGS project: Evolution, critique and future directions. Stat Med. 2009;28:3049-3082. doi:10.1002/sim.3680.

[34] Li P, Vu QD. Identification of parameter correlations for parameter estimation in dynamic biological models. BMC Syst Biol. 2013;7(1):91. doi:10.1186/1752-0509-7-91.

[35] Steuer R, Kurths J, Daub CO, et al. The mutual information: detecting and evaluating dependencies between variables. Bioinformatics. 2002;18:S231-S240. doi:10.1093/bioinformatics/18.suppl-2.s231.

[36] Crosby A W. America's forgotten pandemic: the influenza of 1918. Cambridge, UK: Cambridge University Press; 2003.

[37] Chowell G, Nishiura H, Bettencourt LMA. Comparative estimation of the reproduction number for pandemic influenza from daily case notification data. J R Soc Interface. 2007;4:155-166. doi:10.1098/rsif.2006.0161.

[38] Longini IM Jr., Halloran ME, Nizam A, et al. Containing pandemic influenza with antiviral agents. Am J Epidemiol. 2004;159:623-633. doi:10.1093/aje/kwh092.

[39] World Health Organization. Ebola haemorrhagic fever in Zaire, 1976. Switzerland: World Health Organization; 1978.

[40] Breman JG, Piot P, Johnson KM, et al. The epidemiology of Ebola hemorrhagic fever in Zaire, 1976. http://www.enivd.de/EBOLA/ebola-24.htm. Published 1978. Accessed March 7, 2016.

[41] Camacho A, Kucharski AJ, Funk S, et al. Potential for large outbreaks of Ebola virus disease. Epidemics. 2014;9:70-78. doi:10.1016/j.epidem.2014.09.003.

[42] Shibli M, Gooch S, Lewis HE, et al. Common colds on Tristan da Cunha. The Journal of hygiene. 1971;69(2):255-262. doi:10.1017/s0022172400021483.

[43] Hammond BJ, Tyrrell DA. A mathematical model of common-cold epidemics on Tristan da Cunha. The Journal of hygiene. 1971;69(3):423-433. doi:10.1017/s0022172400021677. 
[44] Wearing HJ, Rohani P, Keeling MJ. Appropriate models for the management of infectious diseases. PLoS Med. 2005;2(7):e174. doi:10.1371/journal.pmed.0020174.

[45] Lloyd AL. Sensitivity of model-based epidemiological parameter estimation to model assumptions. In: Chowell G, Hyman JM, Bettencourt LMA, Castillo-Chavez C, ed. Mathematical and Statistical Estimation Approaches in Epidemiology. Dordrecht: Springer; 2009.

[46] Grenfell BT, Bjørnstad ON, Finkenstädt BF. Dynamics of measles epidemics: scaling noise, determinism, and predictability with the TSIR model. Ecol Monogr. 2002;72:185-202. doi:10.2307/3100024.

[47] Bjørnstad ON, Finkenstädt BF, Grenfell BT. Dynamics of measles epidemics: estimating scaling of transmission rates using a time series SIR model. Ecol Monogr. 2002;72:169-184. doi:10.2307/3100023.

[48] He D, Ionides EL, King AA. Plug-and-play inference for disease dynamics: measles in large and small populations as a case study. J R Soc Interface. 2010;7:271-283. doi:10.1098/rsif.2009.0151.

[49] Mollison D, Din SU. Deterministic and Stochastic Models Variability of Measles Transmission. Math Biosci. 1993;117:144-177. doi:10.1016/0025-5564(93)90021-2.

[50] Brown GD, Oleson JJ, Porter AT. An empirically adjusted approach to reproductive number estimation for stochastic compartmental models: a case study of two Ebola outbreaks. Biometrics. 2016;72:335-343.

[51] Bretó C, He D, Ionides EL, King AA. Time series analysis via mechanistic models. Ann Appl Stat. 2009;3(1):319-348. doi:10.1214/08-aoas201.

[52] Coulson T, Rohani P, Pascual M. Skeletons, noise and population growth: the end of an old debate?. Trends Ecol Evol. 2004;19(7):359-364. doi:10.1016/j.tree.2004.05.008. 
[53] Ranta E, Lundberg P, Kaitala V, Laakso J. Visibility of the environmental noise modulating population dynamics. Proc R Soc Lond B Biol Sci. 2000;267(1455):18511856. doi:10.1098/rspb.2000.1220.

[54] Greenman JV, Benton TG. The amplification of environmental noise in population models: causes and consequences. Am Nat. 2003;161(2):225-239. doi:10.1086/345784. 
Figure 1

$\gamma=0.90$

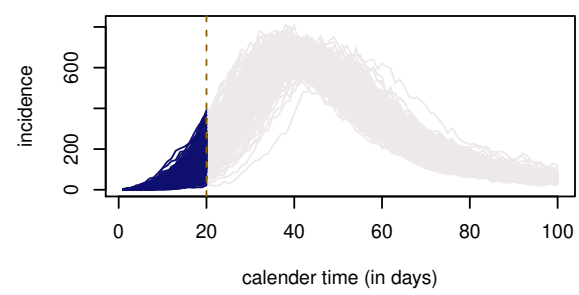

$\gamma=0.94$

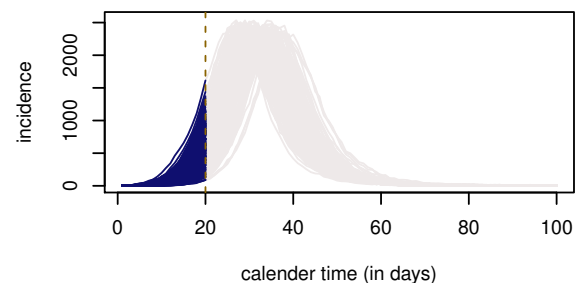

$\gamma=0.98$

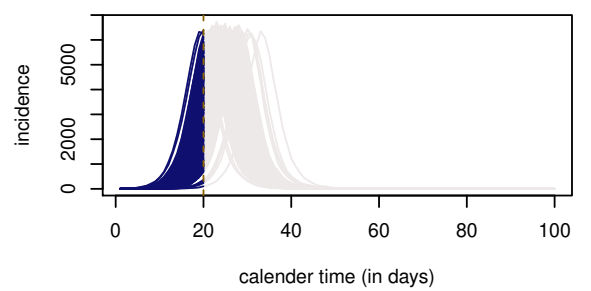

$\gamma=0.92$

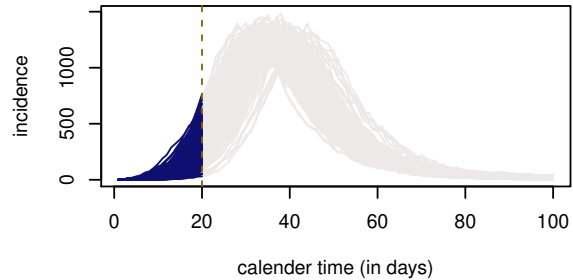

$\gamma=0.96$

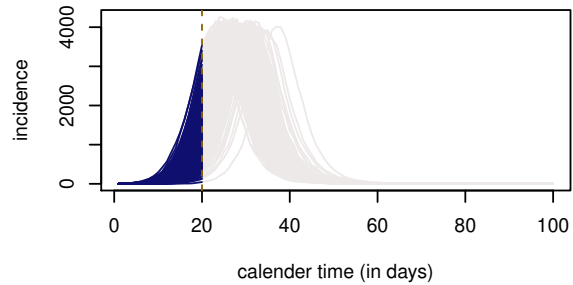

$\gamma=1.00$

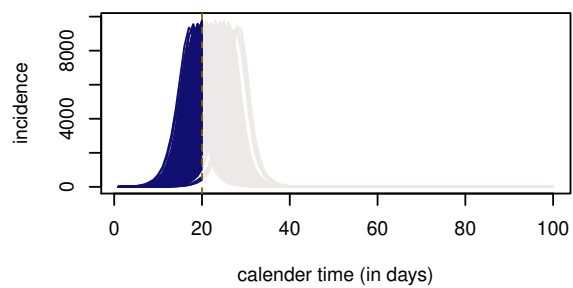

Figure 1: 250 epidemic curve simulations using $N=70,000, \beta=1.1, \alpha=0.4467, I(0)=1$ and different values of $\gamma\{0.90$, $0.92,0.94,0.96,0.98$ and 1.00$\}$. The dark colored part of the curves appearing before the dotted vertical line show the early epidemic growth phase used in the analyses; the light colored part shows the trajectories beyond the early growth phase. The lower the value of $\gamma$, the slower the epidemics take off and the longer they take to reach peak time, and vice versa. 
Figure 2
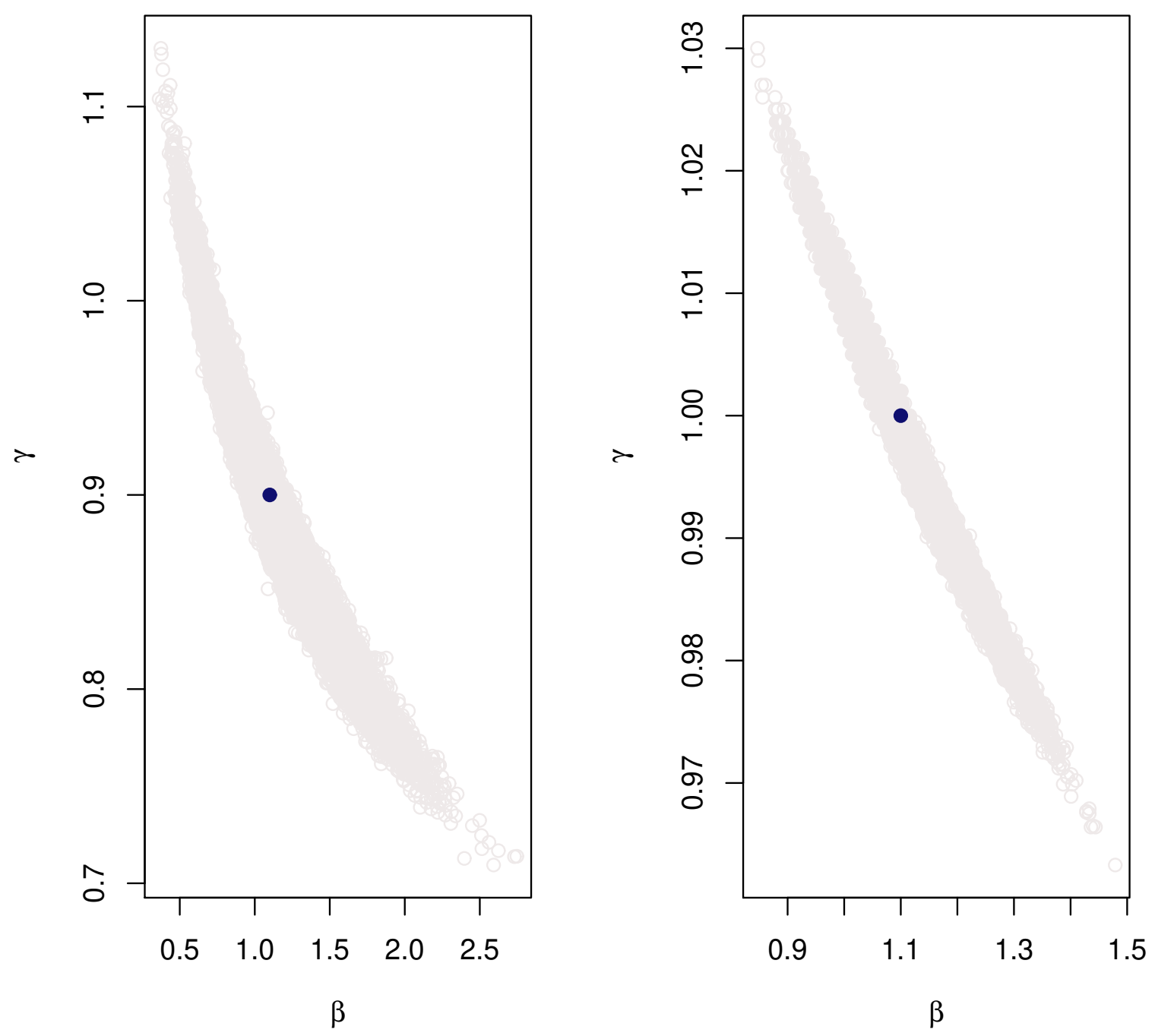

Figure 2: Scatter plot of $\beta$ and $\gamma$ posterior samples (light coloured dots). Left: true value of $\gamma$ is 0.9 ; Right: true value of $\gamma$ is 1.0. The dark coloured dot represents the true values. 


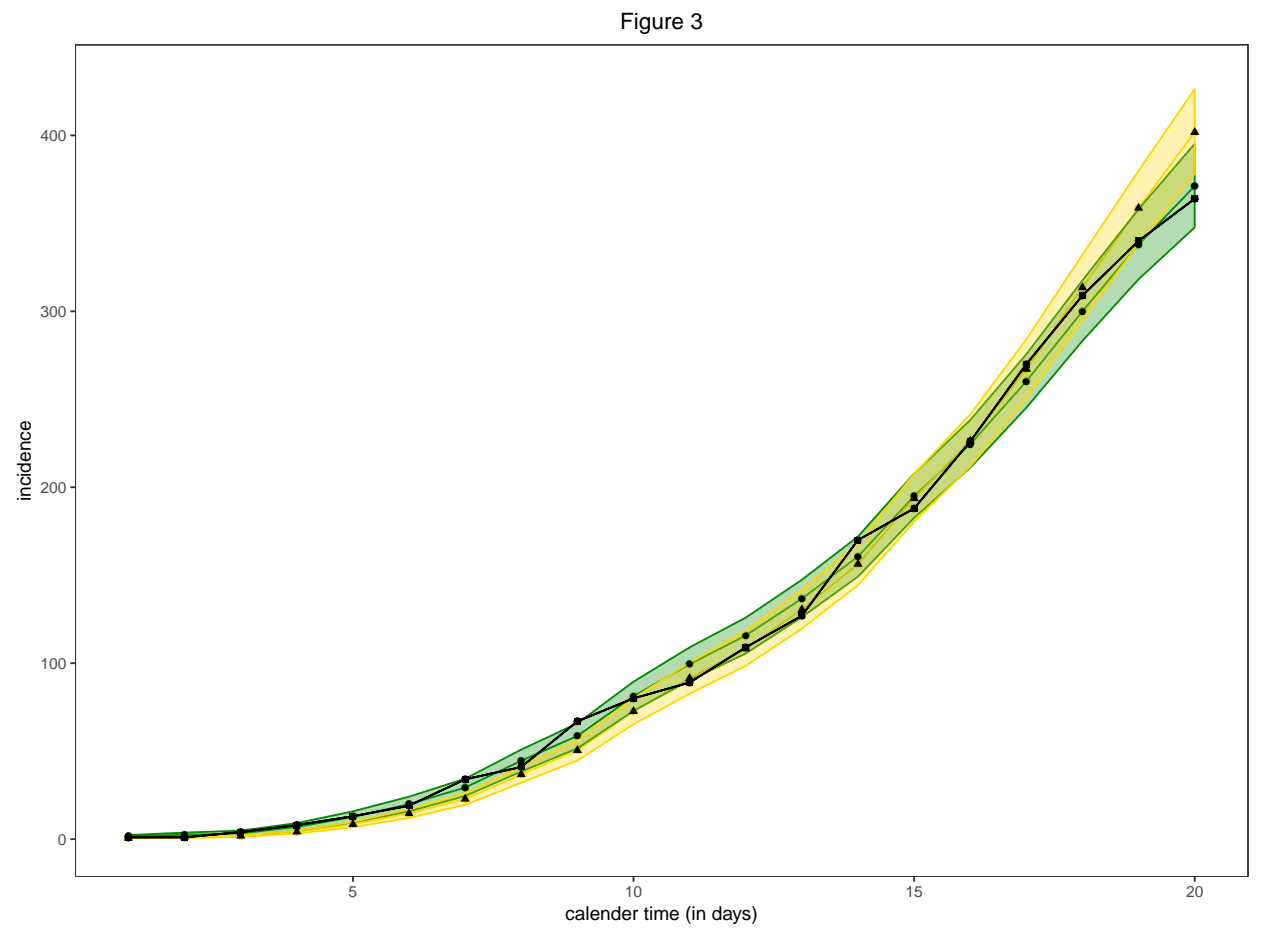

Model - non-linear mass action (MSPE=193) $\triangle$ bi-linear mass action (MSPE=285) $\square$ simulated cases

Figure 3: Non-linear and bi-linear mass action fitted to an epidemic curve simulated with $\gamma=0.9$. Solid lines represent observed data and the fitted models. Shadings represent $95 \%$ credible interval of the fitted models. 


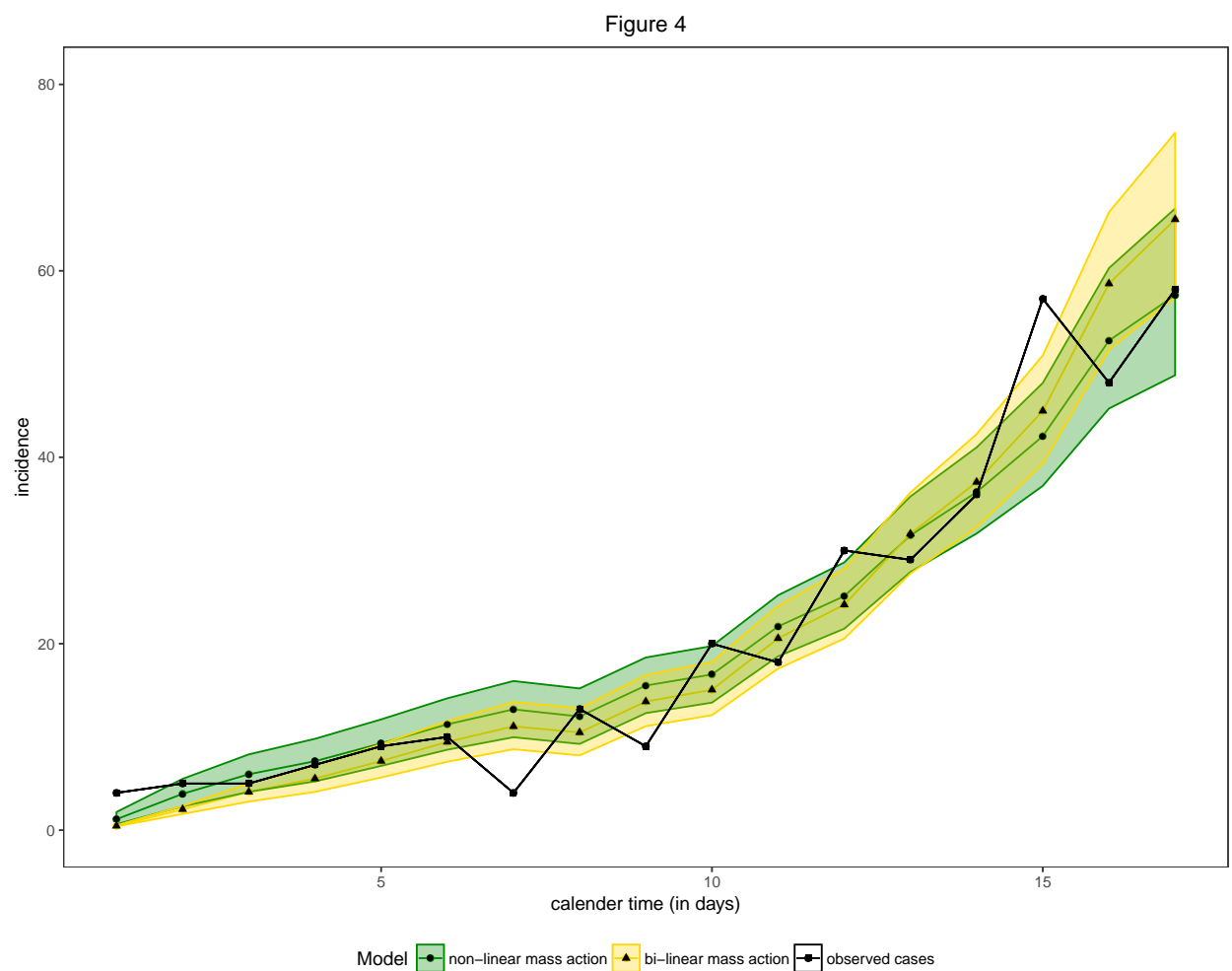

Figure 4: Comparison of fitted vs observed: bi-linear and non-linear mass action. Solid lines represent observed data and the fitted models. Shadings represent $95 \%$ credible interval of the fitted model. 


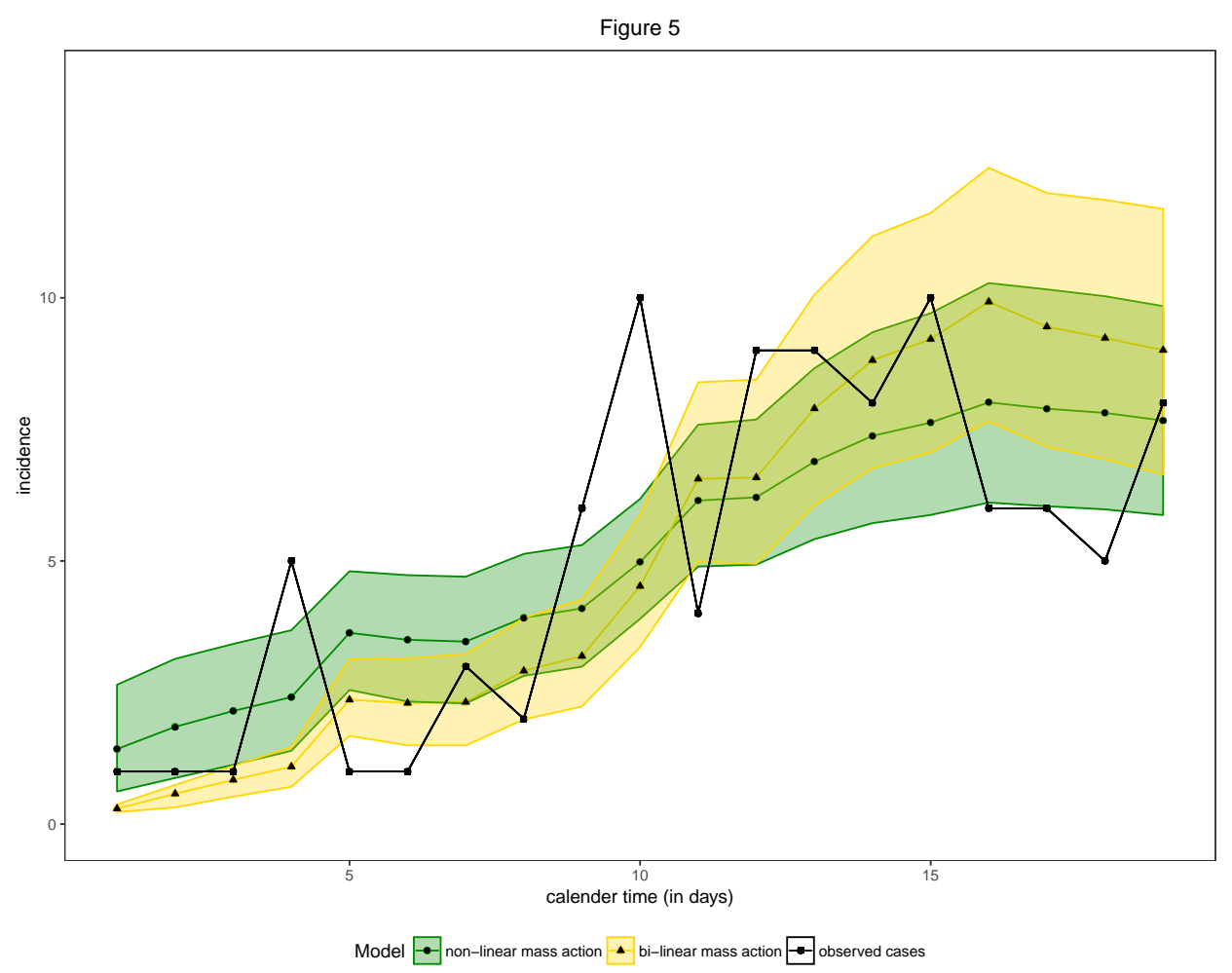

Figure 5: Comparison of fitted vs observed: bi-linear and non-linear mass action. Solid lines represent observed data and the fitted models. Shadings represent $95 \%$ credible interval of the fitted model. 


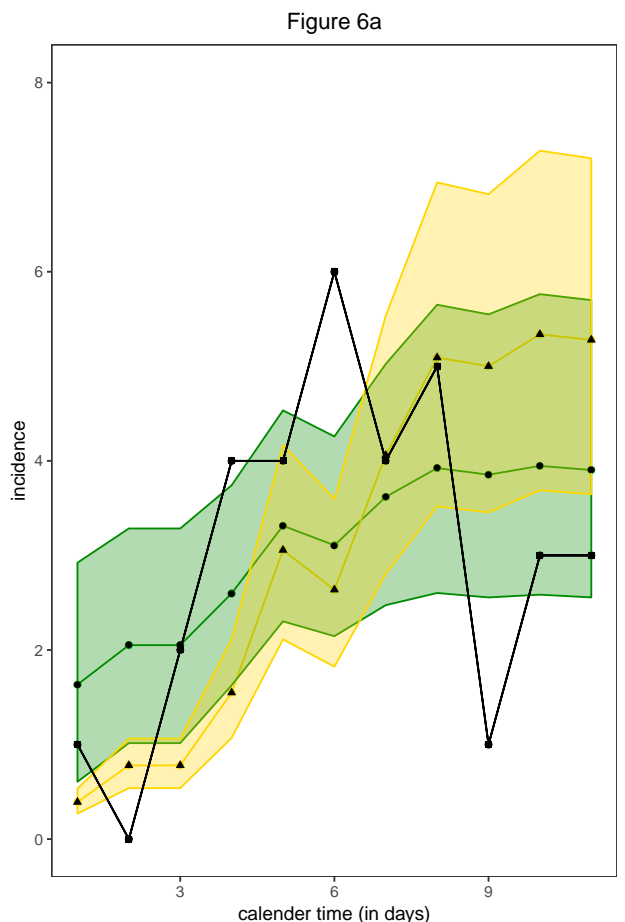

Model $\rightarrow$ non-linear mass action $\triangle$ bi-linear mass action $\rightarrow$ observed cases

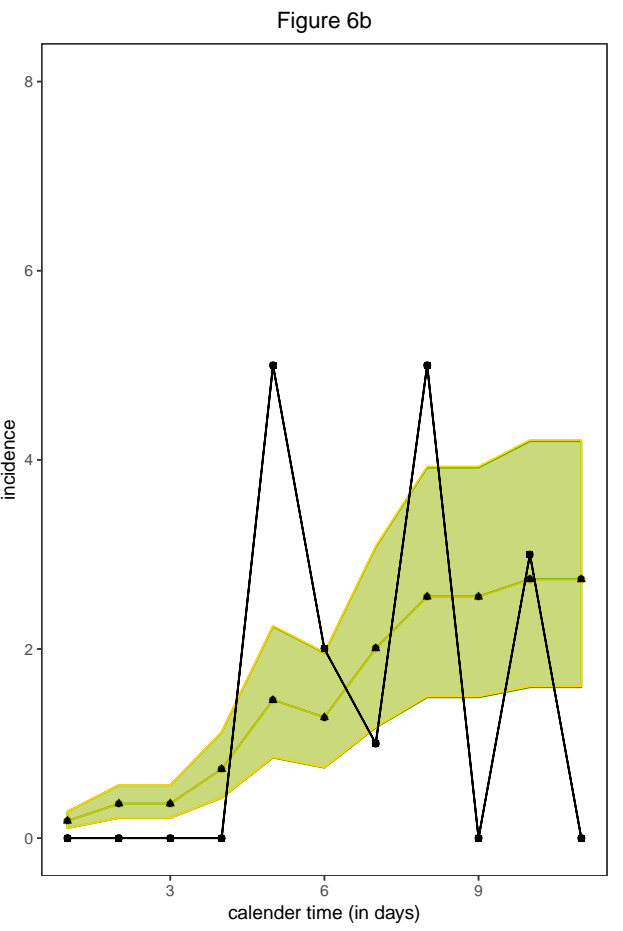

Model $\rightarrow$ non-linear mass action $\triangle$ bi-linear mass action $\square$ observed cases

Figure 6: Comparison of fitted vs observed for $I_{n e w}(t)$ and $R_{n e w}(t)$ : bi-linear and non-linear mass action. Solid lines represent observed data and the fitted models. Dotted lines represent $95 \%$ credible interval of the fitted model. 
Table 1: Simulation summary: The simulation studies estimation of all model parameters simultaneously assuming both $\Delta N_{I}(t)$ and $\Delta N_{R}(t)$ are observed as well as when only $\Delta N_{I}(t)$ is observed with model quantities and parameters set to $N=70,000, T=20$ days, $\beta=1.1, \gamma=0.9, \alpha=0.4467, I(0)=1$ corresponding to $R_{0}=2.46$.

\begin{tabular}{|c|c|c|c|c|c|}
\hline \multirow{2}{*}{$\begin{array}{c}\alpha \\
\text { prior }\end{array}$} & & $\beta$ & $\gamma$ & $\alpha$ & $R_{0}$ \\
\hline & \multicolumn{5}{|c|}{ Estimation using both $\Delta N_{I}(t)$ and $\Delta N_{R}(t)$} \\
\hline \multirow{5}{*}{$\alpha \sim U(0.1,1)$} & coverage $(\%)$ & 85.7724 & 86.9919 & 91.8699 & 90.2439 \\
\hline & bias & 0.1682 & -0.0212 & 0.0038 & 0.2208 \\
\hline & variance & 0.0678 & 0.0018 & 0.0005 & 0.1246 \\
\hline & st.error & 0.2603 & 0.0427 & 0.0234 & 0.3529 \\
\hline & RMSE & 0.3099 & 0.0477 & 0.0237 & 0.4163 \\
\hline \multirow{6}{*}{$\alpha \sim U(0.1,1)$} & \multicolumn{5}{|c|}{ Estimation using $\Delta N_{I}(t)$ only } \\
\hline & coverage $(\%)$ & 94.4000 & 97.2000 & 99.6000 & 99.2000 \\
\hline & bias & 0.1404 & -0.0325 & -0.0478 & 1.2842 \\
\hline & variance & 0.0688 & 0.0023 & 0.0146 & 1.1794 \\
\hline & st.error & 0.2623 & 0.0478 & 0.1207 & 1.0860 \\
\hline & RMSE & 0.2975 & 0.0578 & 0.1298 & 1.6818 \\
\hline \multirow{5}{*}{$\alpha \sim U(0.4,0.5)$} & coverage $(\%)$ & 97.2000 & 96.0000 & 100.0000 & 97.2000 \\
\hline & bias & 0.1419 & -0.0159 & 0.0012 & 0.1971 \\
\hline & variance & 0.0494 & 0.0016 & 0.0000 & 0.1188 \\
\hline & st.error & 0.2223 & 0.0397 & 0.0028 & 0.3447 \\
\hline & RMSE & 0.2637 & 0.0427 & 0.0030 & 0.3971 \\
\hline \multirow{5}{*}{$\alpha=0.4467$} & coverage $(\%)$ & 97.2000 & 96.0000 & \multirow{5}{*}{-} & 97.2000 \\
\hline & bias & 0.1409 & -0.0159 & & 0.1945 \\
\hline & variance & 0.0494 & 0.0016 & & 0.1177 \\
\hline & st.error & 0.2222 & 0.0397 & & 0.3431 \\
\hline & RMSE & 0.2631 & 0.0427 & & 0.3944 \\
\hline
\end{tabular}


Table 2: Simulation summary: the simulation studies simultaneous estimation of $\beta$ and $\gamma$ for different values of $\gamma$ when only $I_{n e w}(t)$ is observed; $\alpha$ is fixed to its true value. In data simulation other model quantities and parameters were set to $N=70,000, T=20$ days, $\beta=1.1, \alpha=0.4467, I(0)=1$ corresponding to $R_{0}=2.46$.

\begin{tabular}{|c|c|c|c|c|c|}
\hline$\gamma$ & property & $\beta$ & $\gamma$ & $R_{0}$ & median MSPE \\
\hline \multirow{5}{*}{0.90} & coverage $(\%)$ & 97.2000 & 96.0000 & 97.2000 & \multirow{5}{*}{110.9000} \\
\hline & bias & 0.1409 & -0.0159 & 0.3154 & \\
\hline & variance & 0.0494 & 0.0016 & 0.2475 & \\
\hline & st.error & 0.2222 & 0.0397 & 0.4975 & \\
\hline & RMSE & 0.2631 & 0.0427 & 0.5891 & \\
\hline \multirow{5}{*}{0.92} & coverage (\%) & 97.6000 & 96.8000 & 97.6000 & \multirow{5}{*}{173.5000} \\
\hline & bias & 0.0831 & -0.0065 & 0.1860 & \\
\hline & variance & 0.0368 & 0.0011 & 0.1843 & \\
\hline & st.error & 0.1917 & 0.0336 & 0.4293 & \\
\hline & RMSE & 0.2090 & 0.0342 & 0.4678 & \\
\hline \multirow{5}{*}{0.94} & coverage & 97.6000 & 97.6000 & 97.6000 & \multirow{5}{*}{287.7000} \\
\hline & bias & 0.0435 & -0.0024 & 0.0974 & \\
\hline & variance & 0.0237 & 0.0006 & 0.1187 & \\
\hline & st.error & 0.1539 & 0.0245 & 0.3445 & \\
\hline & RMSE & 0.1599 & 0.0246 & 0.3580 & \\
\hline \multirow{5}{*}{0.96} & coverage (\%) & 95.6000 & 94.8000 & 95.6000 & \multirow{5}{*}{562.9000} \\
\hline & bias & 0.0219 & 0.0008 & 0.0490 & \\
\hline & variance & 0.0182 & 0.0006 & 0.0910 & \\
\hline & st.error & 0.1348 & 0.0240 & 0.3017 & \\
\hline & RMSE & 0.1365 & 0.0240 & 0.3056 & \\
\hline \multirow{5}{*}{0.98} & coverage & 95.9839 & 96.3855 & 95.9839 & \multirow{5}{*}{1167.0000} \\
\hline & bias & -0.0012 & 0.0019 & -0.0028 & \\
\hline & variance & 0.0097 & 0.0002 & 0.0485 & \\
\hline & st.error & 0.0984 & 0.0133 & 0.2202 & \\
\hline & RMSE & 0.0984 & 0.0135 & 0.2203 & \\
\hline \multirow{5}{*}{1.00} & coverage & 97.2000 & 96.8000 & 97.2000 & \multirow{5}{*}{2703.0000} \\
\hline & bias & -0.0028 & 0.0013 & -0.0062 & \\
\hline & variance & 0.0064 & 0.0001 & 0.0319 & \\
\hline & st.error & 0.0798 & 0.0095 & 0.1787 & \\
\hline & RMSE & 0.0799 & 0.0096 & 0.1788 & \\
\hline
\end{tabular}


Table 3: Summary of simulation results when bi-linear mass action is assumed given that $\gamma=$ $0.90,0.92,0.94,0.96,0.98$ and $1 ; \alpha$ is fixed to its true value. In data simulation other model quantities and parameters were set to $N=70,000, T=20$ days, $\beta=1.1, \alpha=0.4467, I(0)=1$ corresponding to $R_{0}=2.46$.

\begin{tabular}{|c|c|c|c|c|}
\hline scenario & property & $\beta$ & $R_{0}$ & median MSPE \\
\hline \multirow{5}{*}{$\gamma=0.90$} & coverage $(\%)$ & 0.0000 & 0.0000 & \multirow{5}{*}{124.5000} \\
\hline & bias & -0.4304 & -0.9634 & \\
\hline & variance & 0.0008 & 0.0040 & \\
\hline & st.error & 0.0282 & 0.0632 & \\
\hline & RMSE & 0.4313 & 0.9655 & \\
\hline \multirow{5}{*}{$\gamma=0.92$} & coverage $(\%)$ & 0.0000 & 0.0000 & \multirow{5}{*}{193.9000} \\
\hline & bias & -0.3892 & -0.8712 & \\
\hline & variance & 0.0008 & 0.0040 & \\
\hline & st.error & 0.0283 & 0.0634 & \\
\hline & RMSE & 0.3902 & 0.8735 & \\
\hline \multirow{5}{*}{$\gamma=0.94$} & coverage $(\%)$ & 0.0000 & 0.0000 & \multirow{5}{*}{330.9000} \\
\hline & bias & -0.3365 & -0.7534 & \\
\hline & variance & 0.0006 & 0.0031 & \\
\hline & st.error & 0.0247 & 0.0553 & \\
\hline & RMSE & 0.3375 & 0.7554 & \\
\hline \multirow{5}{*}{$\gamma=0.96$} & coverage $(\%)$ & 0.4000 & 0.4000 & \multirow{5}{*}{624.8000} \\
\hline & bias & -0.2597 & -0.5814 & \\
\hline & variance & 0.0006 & 0.0030 & \\
\hline & st.error & 0.0246 & 0.0551 & \\
\hline & RMSE & 0.2609 & 0.5840 & \\
\hline \multirow{5}{*}{$\gamma=0.98$} & coverage $(\%)$ & 0.0000 & 0.0000 & \multirow{5}{*}{1228.0000} \\
\hline & bias & -0.1543 & -0.3453 & \\
\hline & variance & 0.0004 & 0.0018 & \\
\hline & st.error & 0.0188 & 0.0421 & \\
\hline & RMSE & 0.1554 & 0.3479 & \\
\hline \multirow{5}{*}{$\gamma=1.00$} & coverage $(\%)$ & 92.4000 & 92.8000 & \multirow{5}{*}{2678.0000} \\
\hline & bias & -0.0016 & -0.0036 & \\
\hline & variance & 0.0002 & 0.0009 & \\
\hline & st.error & 0.0135 & 0.0302 & \\
\hline & RMSE & 0.0136 & 0.0304 & \\
\hline
\end{tabular}


Table 4: Parameter estimates of bi-linear and non-linear mass action models for the influenza epidemic in the city of San Fransisco. The removal rate $\alpha$ is assigned an informative prior $(U(1 / 6,1 / 3))$.

\begin{tabular}{cccccc}
\hline \multirow{2}{*}{ mass action } & \multicolumn{4}{c}{ posterior mean (HPD interval) } & \multicolumn{2}{c}{ MSPE } \\
& $\beta$ & $\gamma$ & $\alpha$ & $R_{0}$ & \\
\hline bi-linear & $0.486(0.379,0.582)$ & - & $0.273(0.191,0.333)$ & $1.801(1.451,2.210)$ & 54.352 \\
non-linear & $1.173(0.559,1.846)$ & $0.789(0.659,0.923)$ & $0.254(0.176,0.333)$ & $4.748(2.219,7.983)$ & 51.013 \\
\hline
\end{tabular}


Table 5: Parameter estimates of bi-linear and non-linear mass action models for the ebola outbreak in Congo. The removal rate $\alpha$ is assigned an informative prior $(U(1 / 6,1 / 5))$.

\begin{tabular}{cccccc}
\hline \multirow{2}{*}{ mass action } & \multicolumn{4}{c}{ posterior mean (HPD interval) } & \multicolumn{2}{c}{ MSPE } \\
\hline bi-linear & $0.295(0.223,0.372)$ & $\gamma$ & $\alpha$ & $R_{0}$ & \\
non-linear & $1.420(0.525,2.478)$ & $0.496(0.258,0.741)$ & $0.180(0.168,0.191)$ & $1.644(1.245,2.077)$ & 12.080 \\
\hline
\end{tabular}


Table 6: Parameter estimates of bi-linear and non-linear mass action models for the common cold outbreak on the island Tristan da Cunha. The removal rate $\alpha$ is estimated with non-informative prior $(U(0,1))$

\begin{tabular}{cccccc}
\hline \multirow{2}{*}{ mass action } & \multicolumn{2}{c}{ posterior mean (HPD interval) } & \multicolumn{2}{c}{ MSPE } \\
& $\beta$ & $\gamma$ & $\alpha$ & $R_{0}$ & \\
\hline bi-linear & $0.393(0.264,0.533)$ & - & $0.182(0.100,0.270)$ & $2.287(1.112,3.717)$ & 7.969 \\
non-linear & $1.640(0.540,2.818)$ & $0.381(0.100,0.707)$ & $0.183(0.102,0.274)$ & $9.530(2.300,18.090)$ & 6.186 \\
\hline
\end{tabular}

